Supporting Information

\title{
Chameleon-Inspired Variable Coloration Enabled by a Highly Flexible Photonic Cellulose Film
}

Ze-Lian Zhang, Xiu Dong, Yi-Ning Fan, Lu-Ming Yang, Lu-He, Fei Song*, Xiu-Li

Wang, Yu-Zhong Wang*

The Collaborative Innovation Center for Eco-Friendly and Fire-Safety Polymeric Materials, National Engineering Laboratory of Eco-Friendly Polymeric Materials (Sichuan), State Key Laboratory of Polymer Materials Engineering, College of Chemistry, Sichuan University, Chengdu 610064, China.

* Corresponding authors. songfei520@gmail.com (F. Song); yzwang@scu.edu.cn (Y.Z. Wang) 
(a)

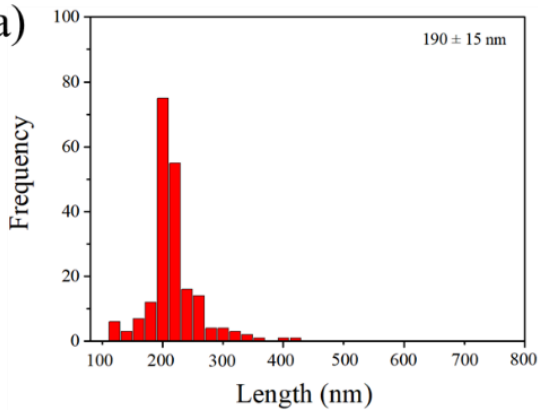

(b)

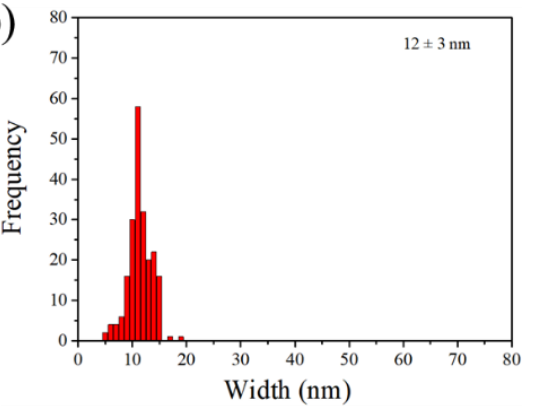

Figure S1. Distribution of (a) length and (b) width of CNC determined from TEM results.
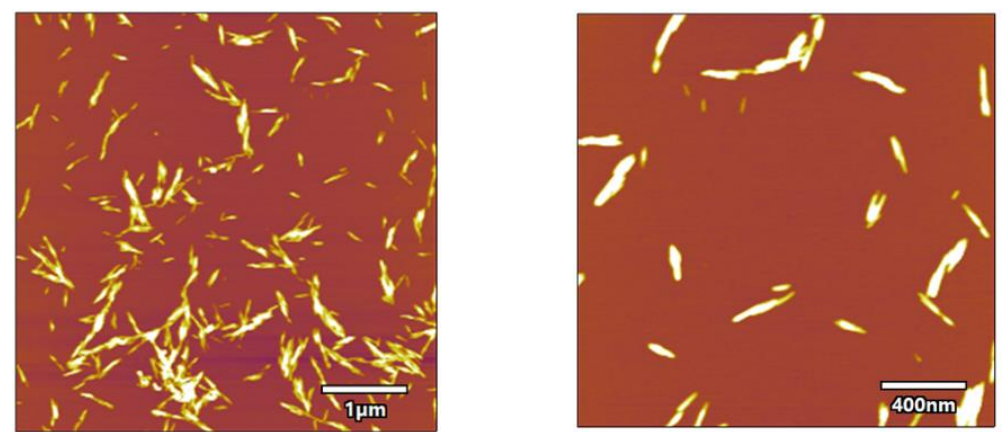

Figure S2. AFM images of CNC at different magnifications.
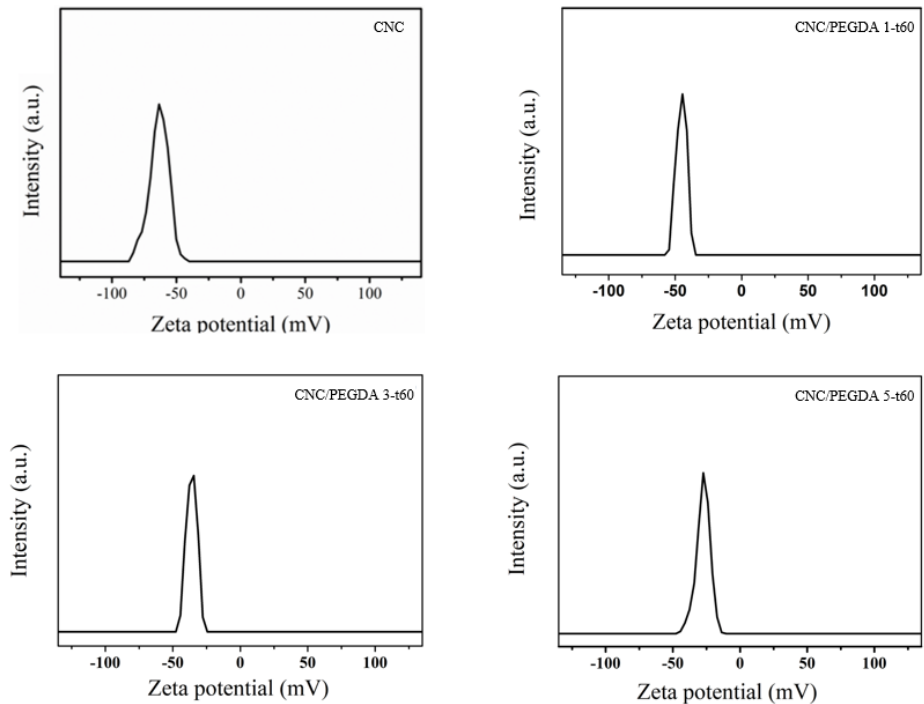

Figure S3. Zeta potential of neat $\mathrm{CNC}$ and $\mathrm{CNC} / \mathrm{PEGDA}$ suspensions containing different PEGDA contents. 


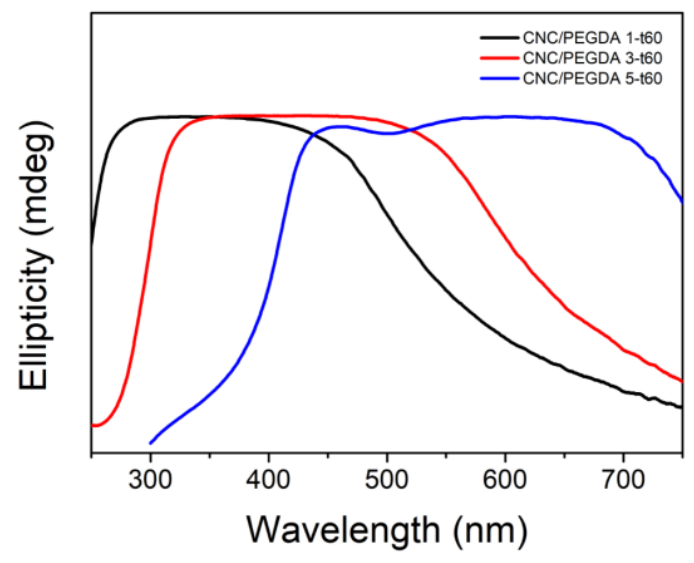

Figure S4. CD spectra of CNC/PEGDA films containing different PEGDA contents.

(a)

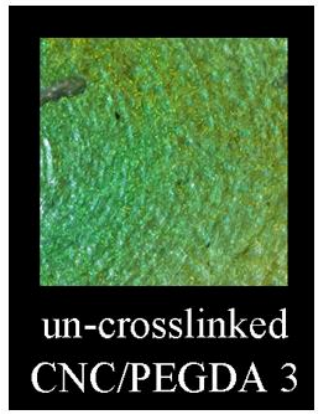

(b)

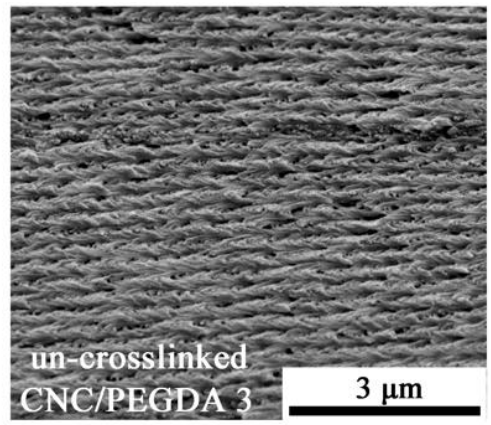

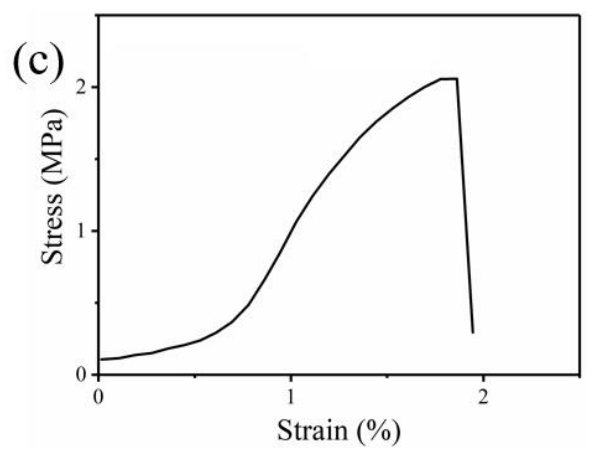

Figure S5. (a) Photographs, (b) SEM images and (c) stress-strain curves of uncrosslinking CNC/PEGDA 3 film.

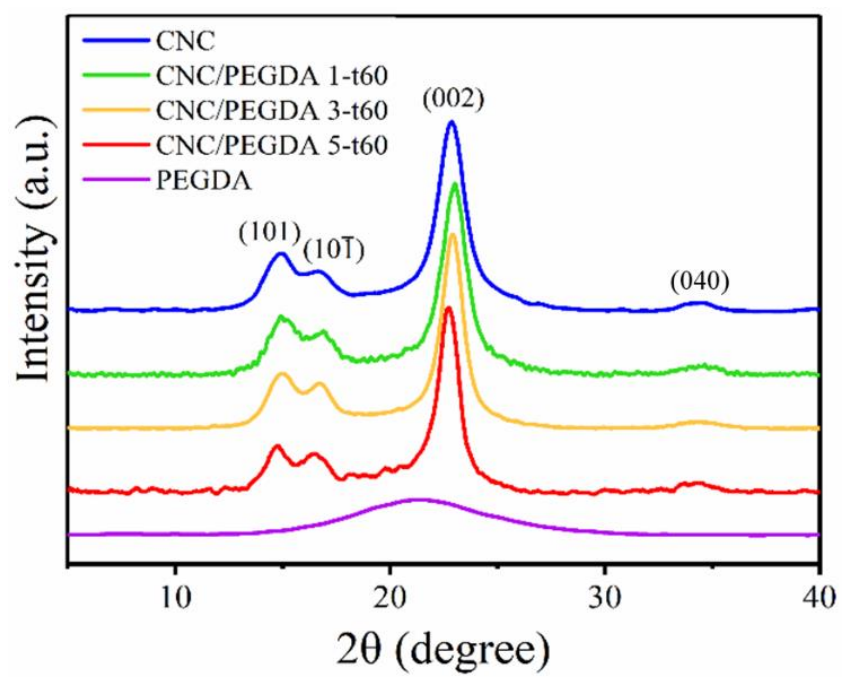

Figure S6. XRD patterns of CNC, CNC/PEGDA films and PEGDA gels. 


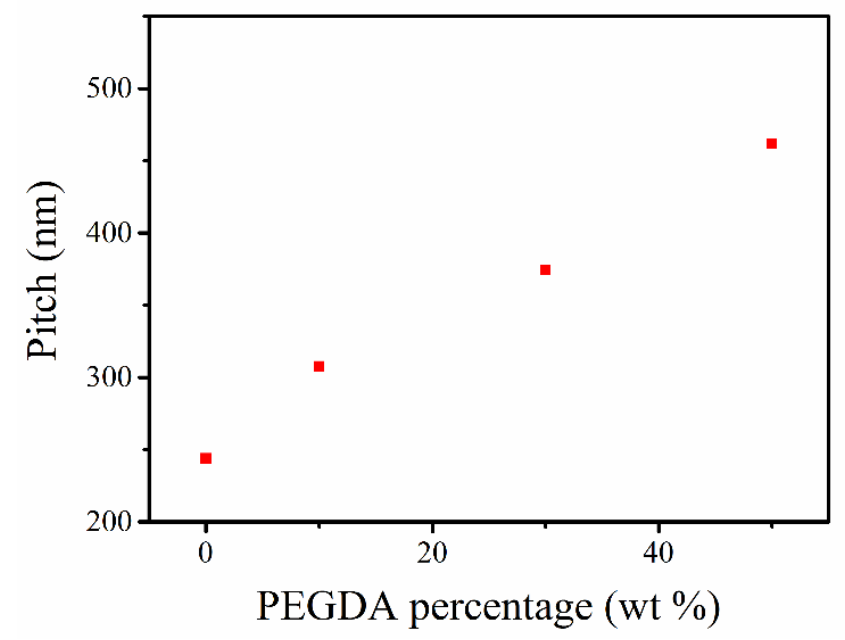

Figure S7. Chiral nematic $P$ of CNC and CNC/PEGDA films calculated from $\lambda_{\max }$ as a function of PEGDA content.

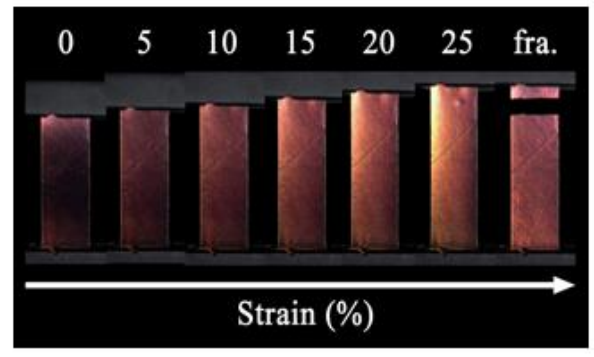

Figure S8. Optical photographs of the CNC/PEGDA 5-t60 film under different stretching strains.
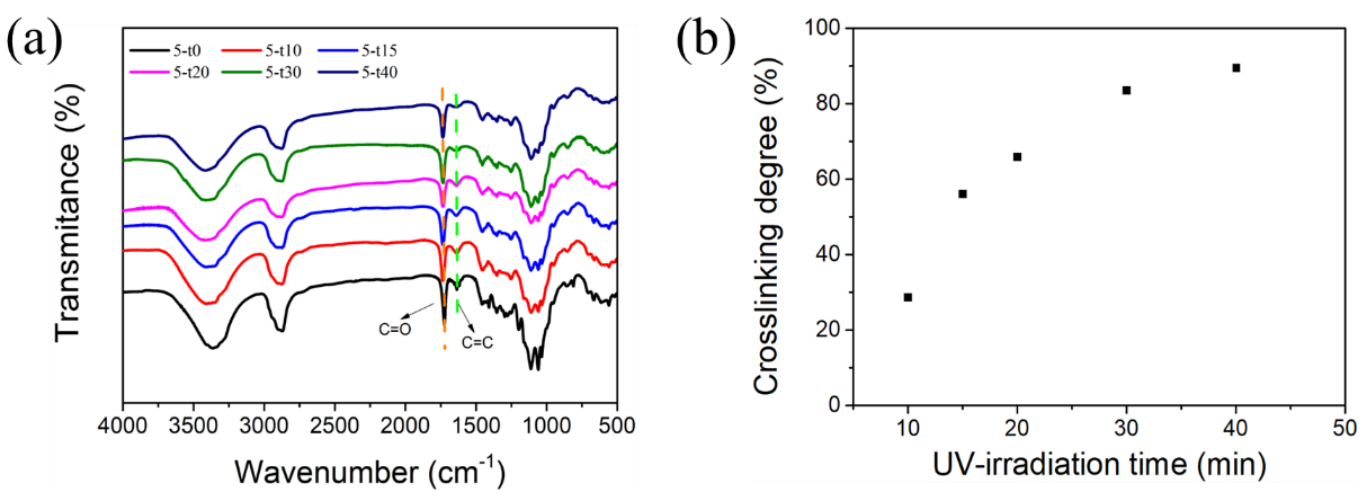

Figure S9. (a) FTIR spectra and (b) crosslinking degree of CNC/PEGDA films prepared at different UV-irradiation times. 


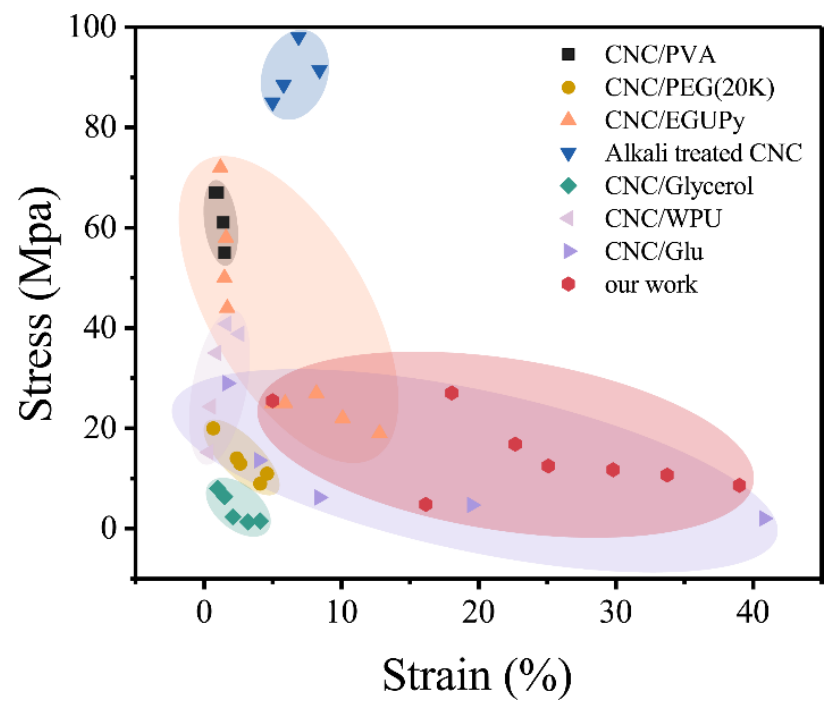

Figure S10. Comparison of tensile strength and strain distribution of iridescent films based on CNCs. $\mathrm{CNCs} / \mathrm{PVA} ;{ }^{1} \mathrm{CNCs} / \mathrm{PEG} ;{ }^{2} \mathrm{CNCs} / \mathrm{EGPUy}$ (a methacrylate derivative with 2-ureido-4pyrimidinone groups); ${ }^{3}$ Alkali treated $\mathrm{CNC}^{4}{ }^{4} \mathrm{CNC} /$ glycerol; ${ }^{5}$ $\mathrm{CNC} / \mathrm{WPU} ;{ }^{6} \mathrm{CNC} /$ glucose. $^{7}$

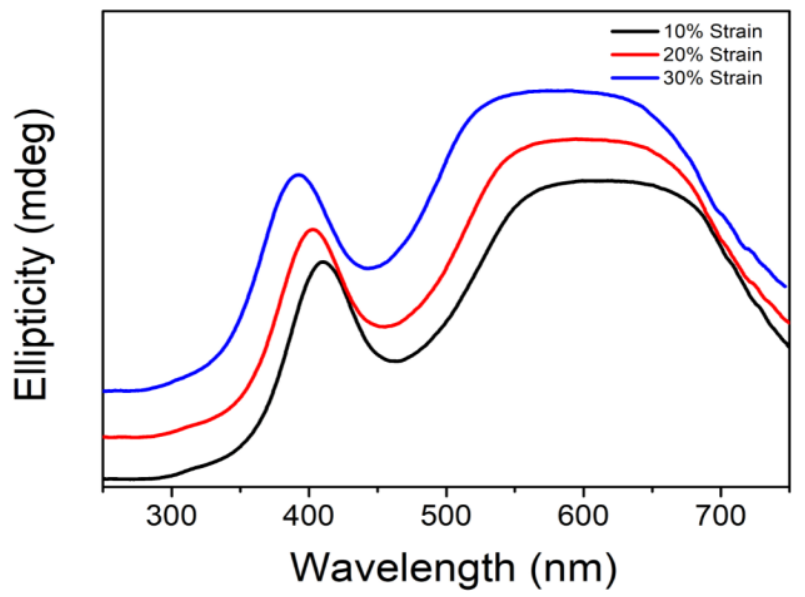

Figure S11. CD spectra of the CNC/PEGDA 5-t15 film upon stretching at different strains. 


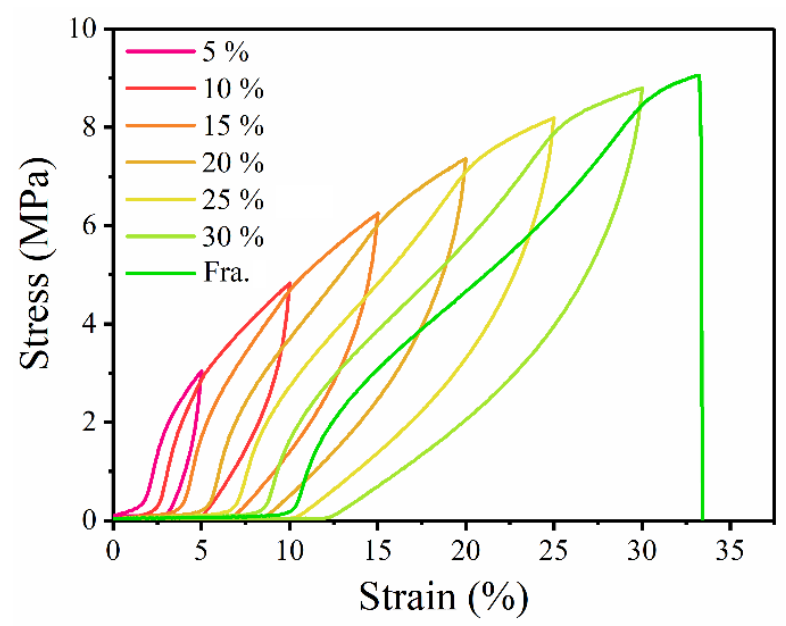

Figure S12. Cyclic tensile loading-unloading curves of CNC/PEGDA 5-t15 film.

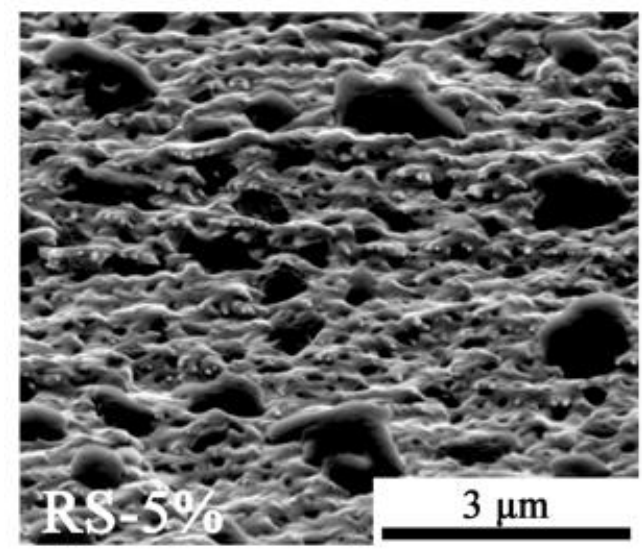

Figure S13. SEM images of CNC/PEGDA 5-t15 film with a residual strain at 5\%.

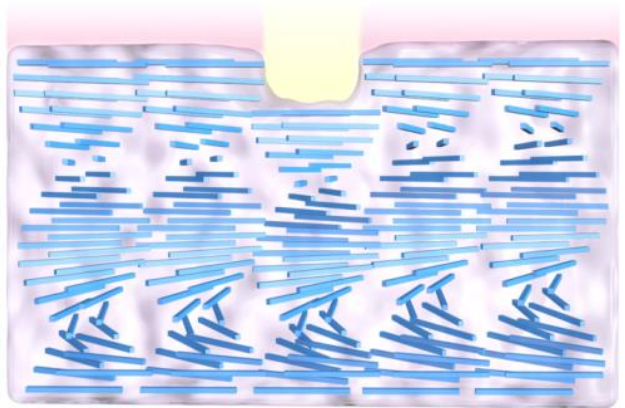

Just written

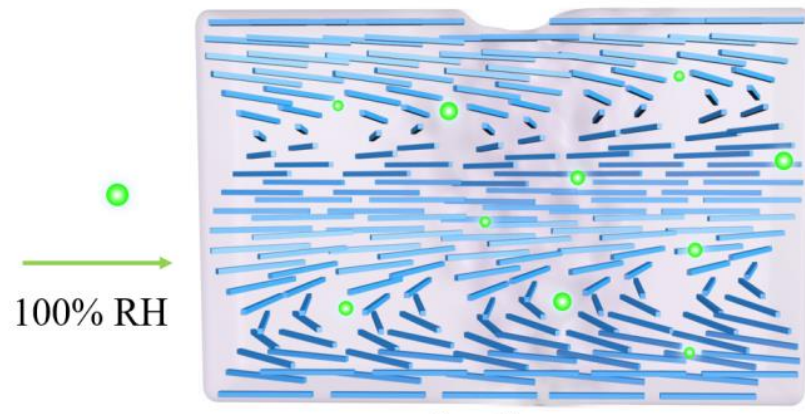

Swollen

Figure S14. Schematic illustration of the humidity-dependent encryption process.

Movie S1. Stretching process of CNC/PEGDA 5-t60 film.

Movie S2. Stretching process of CNC/PEGDA 5-t15 film. 
Movie S3. Reversible color change during cyclic stretching process of CNC/PEGDA

5-t15 film.

\section{REFERENCES}

1. Wang, B. C.; Walther, A. Self-Assembled, Iridescent, Crustacean-Mimetic Nanocomposites with Tailored Periodicity and Layered Cuticular Structure. ACS Nano 2015, 9 (11), 10637-10646.

2. Yao, K.; Meng, Q.; Bulone, V.; Zhou, Q. Flexible and Responsive Chiral Nematic Cellulose Nanocrystal/Poly(ethylene glycol) Composite Films with Uniform and Tunable Structural Color. Adv. Mater. 2017, 29 (28), 1701323.

3. Zhu, B.; Merindol, R.; Benitez, A. J.; Wang, B.; Walther, A. Supramolecular Engineering of Hierarchically Self-Assembled, Bioinspired, Cholesteric Nanocomposites Formed by Cellulose Nanocrystals and Polymers. ACS Appl. Mater. Interfaces 2016, 8 (17), 11031-11040.

4. Nan, F.; Nagarajan, S.; Chen, Y.; Liu, P.; Duan, Y.; Men, Y.; Zhang, J. Enhanced Toughness and Thermal Stability of Cellulose Nanocrystal Iridescent Films by Alkali Treatment. ACS Sustainable Chem. Eng. 2017, 5 (10), 8951-8958.

5. He, Y. D.; Zhang, Z. L.; Xue, J.; Wang, X. H.; Song, F.; Wang, X. L.; Zhu, L. L.; Wang, Y. Z. Biomimetic Optical Cellulose Nanocrystal Films with Controllable Iridescent Color and Environmental Stimuli-Responsive Chromism. ACS Appl. Mater. Interfaces 2018, 10 (6), 58055811.

6. Wan, H.; Li, X. F.; Zhang, L.; Li, X. P.; Liu, P. F.; Jiang, Z. G.; Yu, Z. Z. Rapidly Responsive and Flexible Chiral Nematic Cellulose Nanocrystal Composites as Multifunctional Rewritable Photonic Papers with Eco-Friendly Inks. ACS Appl. Mater. Interfaces 2018, 10 (6), 5918-5925.

7. Qu, D.; Zheng, H.; Jiang, H.; Xu, Y.; Tang, Z. Chiral Photonic Cellulose Films Enabling Mechano/Chemo Responsive Selective Reflection of Circularly Polarized Light. Adv. Opt. Mater. 2019, 7 (7), 1801395. 